\title{
Comparative Analysis of E-AOMDV and MC-AOMDV using Multi-criteria Multipath Routing
}

\author{
Navjot Kaur \\ Student, Dept. of CSE \\ Amritsar College of Engineering \& Technology, \\ PTU, Jalandhar, Punjab.
}

\author{
Tanupreet Singh \\ Professor and HOD, Dept. of ECE \\ Amritsar College of Engineering \& Technology, \\ PTU, Jalandhar, Punjab.
}

\begin{abstract}
In recent decades, numerous attempts have been made on routing the data in mobile ad hoc networks efficiently. Subsequently, several specialists have provided distinct routing protocol for ad hoc networks, especially routing protocols using the concept of multiple paths such as AOMDV. Carefully designed multipath routing protocols promise congestion avoidance, load balancing, improved throughput, fast recovery from link failures hence a more robust network, and a better utilization of the network resources. Various multi path routing protocols have been proposed with aim to minimize the energy utilization in mobile ad hoc networks such as Enhanced Energy Efficient AOMDV. The paper enlightens the critical issue of lowering the routing overhead while preserving the energy consumption in mobile ad hoc networks. The paper presents routing scheme where ratio of residual energy of the nodes and distance is taken into account while making multiple paths between source and destination and further presents a comparison of performance of Enhanced AOMDV and proposed approach.
\end{abstract}

\section{Keywords}

Mobile ad hoc network, AOMDV, E-AOMDV, multi path routing.

\section{INTRODUCTION}

In recent years, significant attention has been paid in bringing out a change in scenario from fixed protocol paradigm to a more adaptive network paradigm for realizing better management and efficiency. Recently MANETs are gaining prominence due to its versatility, simplicity and extremely moderate and cost sparing establishment. Mobile Ad-Hoc Networks are autonomous and decentralized wireless systems comprising of portable nodes which can openly move inside and outside the system. These nodes can act as host/router or both at the same time. They can structure self-assertive topologies relying upon their connectivity with each other in the system. These nodes possess a unique ability to arrange themselves, due to which they can be arranged essentially without the need of any basic infrastructure. The routing is the most active research field in the MANET. The routing protocols designed for wired networks are not suitable for wireless networks due to the node mobility issues in wireless networks. Hence, different protocols are proposed to deal with routing problem in the MANET with the aim to minimize delay, amplify the network throughput, maximize network lifetime and maximize energy efficiency. Energy efficient routing is the most important design criteria for MANETs hence many research efforts have been devoted to developing energy-aware routing protocols.

Routing in mobile ad hoc networks use multiple-hop routing protocols which are able to maintain paths to other nodes and in most cases handle changes in paths due to mobility. The basic principle behind multipath routing protocol is to provide for load balancing in the network. Energy inefficient routing protocol incurs node failure in the network. Since a packet travels in a network in a multi-hop fashion, it is imperative to keep a mobile node operative as long as possible. With an effort to conserve more energy of the network and hence increase its lifetime, energy efficient AOMDV, (E-AOMDV) [5] has also been proposed. This routing protocol takes energy of the nodes into account while forming the multiple paths between the sources to destination node.

\section{RELATED WORK}

Bhavna Sharma. et al. [5], proposed an energy efficient AOMDV in which the nodes which come in path from source to destination are selected on the basis of the residual energy. The nodes are required to broadcast their energy levels to their neighbors so that each node is aware of its surroundings. They have compared the performance of energy efficient AOMDV with AOMDV. EAOMDV has proved to efficiently distribute the load in the network.

Geeta G. et al. [12], proposed an efficient energy based Stable Multipath Routing Scheme aims to reduce energy consumption and provide better stability using the stability model. The proposed scheme consists of three phases like multipath routing, stability of multipath and energy consumption model. Multipath routing is developed to ensure better network lifetime and more energy efficiency. The multipath routing stability is calculated to ensure more network stability. Energy spent for transmission is reduced using the energy consumption model.

Sanjeev Sharma, et al. [33] discussed and compared various protocols in terms of their characteristics, functionality, benefits and limitations. The paper aims to find ways to improve the performance of the protocols. The limited battery energy is an important consideration in the effective operation of an Ad- hoc network. A protocol named as Energy Saving Geographic Routing protocol (ESGRP) using with GPS has been proposed that provides a lower energy cost effective routing solution. ESGRP performs much better in packet delivery owing to selection of better routes using acyclic graph.

M. Bheemalingaiah, et al. [16] proposed a new protocol called Energy-aware Node-disjoint Multipath Routing (ENDMR) protocol, which balances node energy utilization to increase the network lifetime. It takes network congestion into account to reduce the routing delay and increases the reliability of the packets reaching the destination. The proposed protocol significantly reduces the total number of route request packets, hence resulting into an increased packet delivery ratio, decreasing end-to-end delays for the data packets, lower control overhead, fewer collisions of packets, supporting reliability and decreasing power consumption.

Vipul Maheshwari [37] surveyed on different reactive routing protocols of MANET and aimed to enhance the Ad hoc Ondemand Multipath Distance Vector (AODV) routing protocol for MANETs as a delay-aware multi-path protocol. The paper focuses to reduce the frequency of route discovery providing resilience that is especially helpful in scenarios with node mobility in WSN and to improve the QoS in MANETs to 
improve the reliability of data communication and prolong the network lifetime.

Deeptanoy Ghosh, et al. [8] discussed different on-demand routing techniques with their advantages and disadvantages. This paper discusses the need and specialty of routing protocols and the routing challenges in MANET. The different on-demand (reactive) protocols like DSR, AODV, TORA, ABR, DYMO, LMR, LAR, SSA, CBRP, RDMAR, MSR, AOMDV, ARA are studied and compared with their pros and cons, in this paper. And it has found that all these reactive protocols have their own advantages and disadvantages depending upon the situation of the network.

Pinki Nayak, et al. [26] addresses the issue of energy efficient routing to increase the lifetime of the network and discusses a new energy aware routing (EAR) scheme which uses variable transmission range, minimizing the energy consumption at the nodes, thus maximizing the network lifetime. Simulation results show that EAR shows superior performance as compared to common range AODV in terms of energy consumption and improves network lifetime.

Deepthy J, et.al [9] explains that there exists the need for an energy aware and adaptive routing protocol. Noise and interference usually arise while designing a new routing protocol. The proposed solution attempts to make necessary changes to AODV through a cross layer approach. It introduces SINR parameter to take care of reducing interference from other nodes in a high traffic condition. Few parameters such as signal strength, residual battery capacity and queue length have to be monitored before processing a packet to obtain energy efficient routing.

Alpesh Chauhan, et.al [2], proposed different techniques for improved energy function in case of AOMDV. In mobile communication networks, the wireless devices are portable and battery powered, so they have to work in extreme energy constrains. So energy efficiency is the one of the major issues in the mobile networks. This paper has proposed various techniques to help in improving the energy function, such as Minimum Battery Cost Routing (MBCR), Min-Max Battery Cost Routing (MMBCR), Minimum Total Transmission Power Routing (MTPR), Conditional Min-Max Battery Cost Routing (CMMBCR), Min-Max Residual Energy in AOMDV (MMREAOMDV).

\section{ENERGY EFFICIENCY IN MANETS}

As MANETs are a collection of nodes composing a spontaneous, temporary network without any centralized administration or any form of infrastructure where mobile nodes are expected to rely on portable and very limited power sources, each node suffers function halt with the battery drop. Therefore, designing energy efficient multicast routing protocols is an important issue for MANETs.

Energy efficient AOMDV routing protocol is an improved version of AOMDV protocol. AOMDV protocol works on multipath but do not take into consideration the energy of the nodes and the amount of traffic sent through the different paths. While enhanced energy efficient AOMDV includes energy conservation, shortest path and load balancing. In AOMDV, due to imbalanced distribution of load, the nodes with less energy may die soon because they are heavily used in forwarding packets. This leads to imbalanced energy consumption. In Enhanced AOMDV, while selecting one route from multiple routes, energy left at neighbor node is considered and for this each node in the network reports its energy level to its neighbor. In multipath selection, all the next hops from available path are taken in account along with their normalized energy levels and the hop with maximum energy is chosen. Depending upon the energy level of the nodes the load is being distributed. Enhanced AOMDV helps in reducing energy consumption and increase in energy utilization.

\section{MULTIPATH ROUTING PROTOCOL}

Multipath routing is proposed as an alternative to single shortest path routing to distribute load and alleviate congestion in the network. In multipath routing, traffic bound to a destination is split across multiple paths to that destination. Multipath routing aims to establish multiple paths between source-destination pairs and thus requires more hosts to be responsible for the routing tasks. On-demand routing protocols for MANETs discover a route when a source needs to communicate with a destination. The multi-path routing protocol discovers multiple paths during the single route discovery process.

Ad-hoc On-demand Multipath Distance Vector Routing (AOMDV) protocol is an extension to the AODV protocol. Similar to AODV, AOMDV is also based on distance vector concept and uses hop by hop routing approach. AOMDV use route discovery procedures likewise to discover routes on demand. AOMDV achieves path disjointedness by only considering disjoint nodes in all the paths. For route discovery purpose, Route Request packets are proliferated throughout the network which leads to multiple paths at destination node and at the intermediate nodes. Multiples Loop-Free paths are achieved using the advertised hop count method at each node which is required to be maintained at each node in the route table entry. The route entry table at each node further contains a list of next hop along with the corresponding hop counts. This way, every node maintains an advertised hop count for the destination.

The point of interest of using AOMDV is that it facilitates intermediate nodes to reply to RREQs, while still selecting disjoint paths. Due to increased flooding, AOMDV still incurs more message overheads during route discovery and since it is a multipath routing protocol, the destination replies to the multiple RREQs which ultimately results in longer overhead.

\section{PROBLEM FORMULATION}

Due to emerging application requirements, energy efficiency in routing protocols has become the hot topic of research. It plays a very important role in the battery operated ad hoc networks. Energy consumption can be due to receiving the data, transmitting the data, traffic, mobility and size of the network. There is a continuous need to study the energy consumption from a routing overhead point of view.

Each node has the functionality of acting as a router along with being a source or destination. Thus the failure of some nodes operation can greatly impede performance of the network and even affect the basic availability of the network, i.e., routing, availability, etc.

It is paramount important to consider the energy efficiency of while designing a MANET protocol while multipath routing protocol such as AOMDV provides for load balancing in the network, the improved version of this, EAOMDV, strives to preserve the energy of the network by considering the nodes having highest residual energy levels while forming the multiple route from source to destination node. However, this energy efficiency is being achieved at the cost of routing overhead which tends to increase while informing the nodes about energy levels of the nodes. There arises a need to design a protocol in such a way that routing overhead becomes lower while the energy efficiency of the network is being preserved. 
In AOMDV the source sends the data to the destination using multiple paths. This provides load balancing in the network as the nodes in the single path are not used over and over again instead multi path approach allows the load to be distributed among the other nodes. However, to make it more energy efficient the nodes in the path which have more residual energy are chosen so that the network works for longer duration of time. This method required the each node to know about the remaining energy levels of the neighbors, for which the protocol began with the initial steps of broadcasting the messages informing the nodes about residual energy. However, this tends to increase the overhead in the network. In our proposed work, we tend to increase this routing overhead while keeping the multipath routing energy efficient.

\section{PROPOSED APPROACH}

\subsection{Proposed Method}

The proposed approach particularly aims at reducing the broadcasting and the eventual overheads. The whole approach starts with the division of the whole network into the various grids. An initiator is chosen for each grid and the information about the grids is kept with the respective initiator. The division of network into grids and the concept of initiator avoids the initial broadcasting required in the energy efficient AOMDV as all the information required by the nodes to make optimal path is stored with initiator which will provide the source with required knowledge about the neighbor whenever the source has to send data. The path from the source to destination should be shorter in distance as well as the nodes must have higher amount of residual energy. So the ratio of remaining residual energy to the Euclidean distance with the destination tends to be higher for any node to be chosen in the paths.

\subsection{Methodology}

The proposed scheme works on the basis of following steps:

Step 1. First the nodes are deployed in the network.

Step 2. All the network is divided into the grids.

Step 3. Choose one initiator for each grid on the basis of the energy level.

Step 4. Each node of the grid forwards its remaining residual energy level to the initiator along with its location information.

Step 5. The initiator calculates the required ratio of remaining residual energy to the Euclidean distance to the destination for each node.

Step 6. The source node asks the initiator for the nodes with higher ratio so that it can have route to destination.

Step 7. The initiator provides with all the relevant information to form multiple paths.

Step 8. Data is sent using the best optimal paths.

The proposed methodology is needed to be implemented in a tool. The tool opted for simulation of the proposed work is NS2.35.

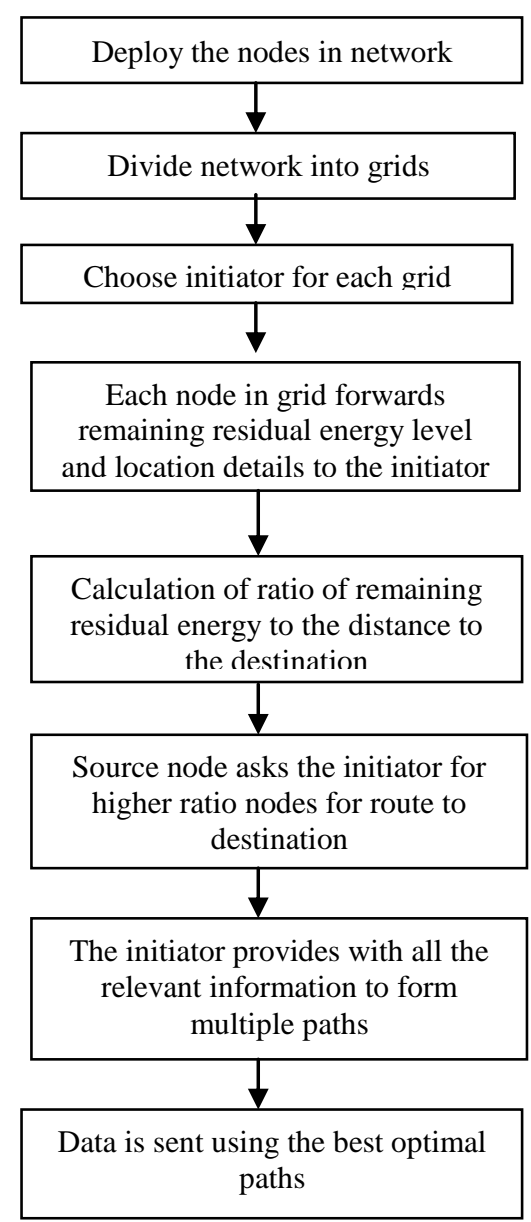

Fig 1: Methodology for proposed scheme

\section{SIMULATION ENVIRONMENT}

In this section, the proposed method has been simulated in Network Simulator 2 (NS2.35) and the simulation results are presented. The operating system is windows 7. NS2 is not supported by windows environment so VMware workstation is installed to provide the virtual environment for the installation of Ubuntu. The simulation parameters are that are used for experiment are listed in Table 1.

Table 1: Simulation Parameters

\begin{tabular}{|c|c|}
\hline Parameter & Value \\
\hline Simulator & NS2.35 \\
\hline Channel & Wireless Channel \\
\hline Propagation Model & 50 \\
\hline No. of nodes & 1000m X 1000m \\
\hline Dimensions of Simulated Area & Drop Tail \\
\hline Queue & Omni-Directional \\
\hline Antenna & AOMDV \\
\hline Routing Protocol & Radio Energy Model \\
\hline Energy Model & 100J \\
\hline Initial Energy &
\end{tabular}




\subsection{Performance metrics}

The performance of the proposed approach has been measured according to four metrics:

1) Energy Consumption: The energy consumption in the network reflects the lifetime. The network which consumes more energy has lesser lifetime.

2) Throughput: The amount of data which is being received by the destination is shown by the Throughput.

3) Average end-to-end delay: The data which is being sent by the source must reach the destination at the earliest. End- toend delay represents the time it takes for the data to reach the destination node.

4) Routing packet overhead: Routing packets overhead is measure of the link quality in the network. If there is link breakage then more number of routing packets needs to be sent to maintain the route. Lesser the overhead, better the network.

\section{SIMULATION RESULTS}

Following are the simulation results of proposed scheme based upon above stated performance metrics.

\subsection{Average Energy Consumption}

Average Energy consumption is defined as the average amount of energy consumed in the network.

The graph in Fig 2 shows the comparison of the energy consumed in EAOMDV and Proposed scheme. Initially 100 joules of the energy was supplied to the network and after the data is sent through selected path, it was observed that energy consumed in EAOMDV is approximately same in comparison to energy consumed in proposed work. This means that energy efficiency is achieved in the network.

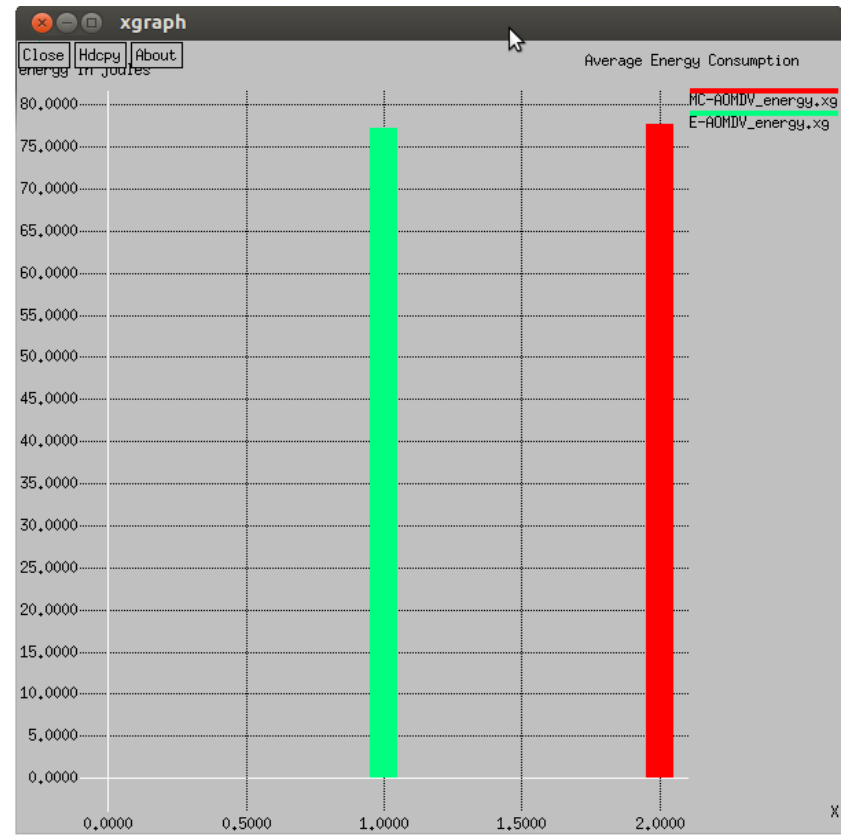

Fig. 2: Average Remaining Energy Comparison of EAOMDV and MC-AOMDV

\subsection{Throughput}

Throughput is defined as the ratio of number of data packets sent or received per unit time of the network.
Graph in Fig. 3 shows the throughput comparison between proposed method and E-AOMDV. Since throughput in EAOMDV is $95 \mathrm{Kbps}$ and is $132 \mathrm{Kbps}$ in Proposed scheme (MCAOMDV), so our work is found to be better than E-AOMDV.

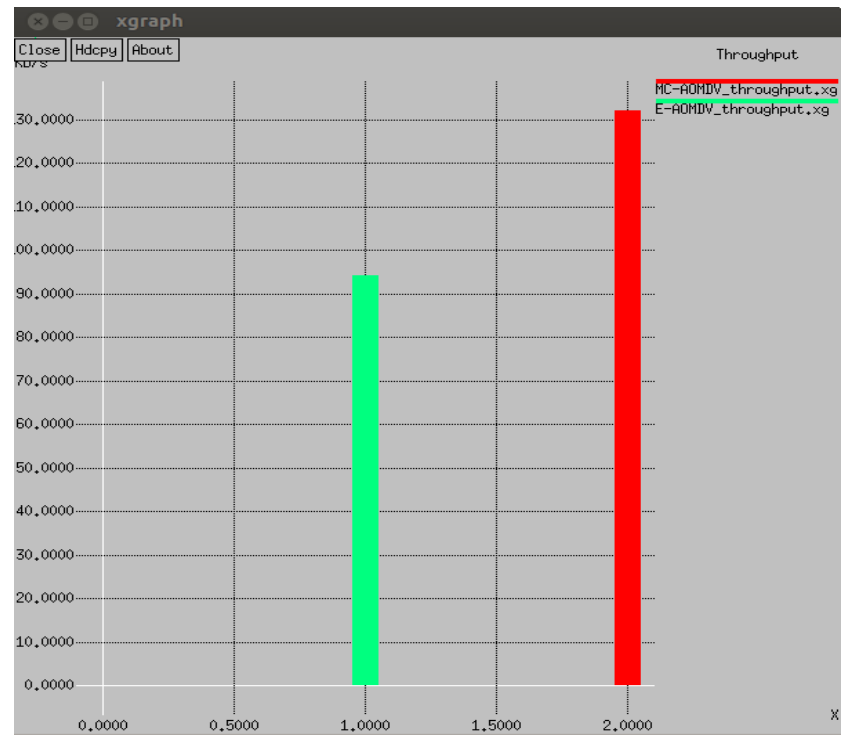

Fig 3: Throughput Comparison of E-AOMDV and MCAOMDV

\subsection{Average End-to-End Delay}

Average end-to-end delay or latency is defined as the average time taken by the data packets to reach from source to destination.

The graph in Fig 4 shows the delay comparison. Lesser the delay, better the network's performance. Proposed scheme (MCAOMDV) has lesser delay as compared to E-AOMDV. This resaon is primarily beacuse the nodes have to mutually broadcasts the remaining energy levels to their neighbors which tends to consume a lot of time before path could be established from source to destination node.

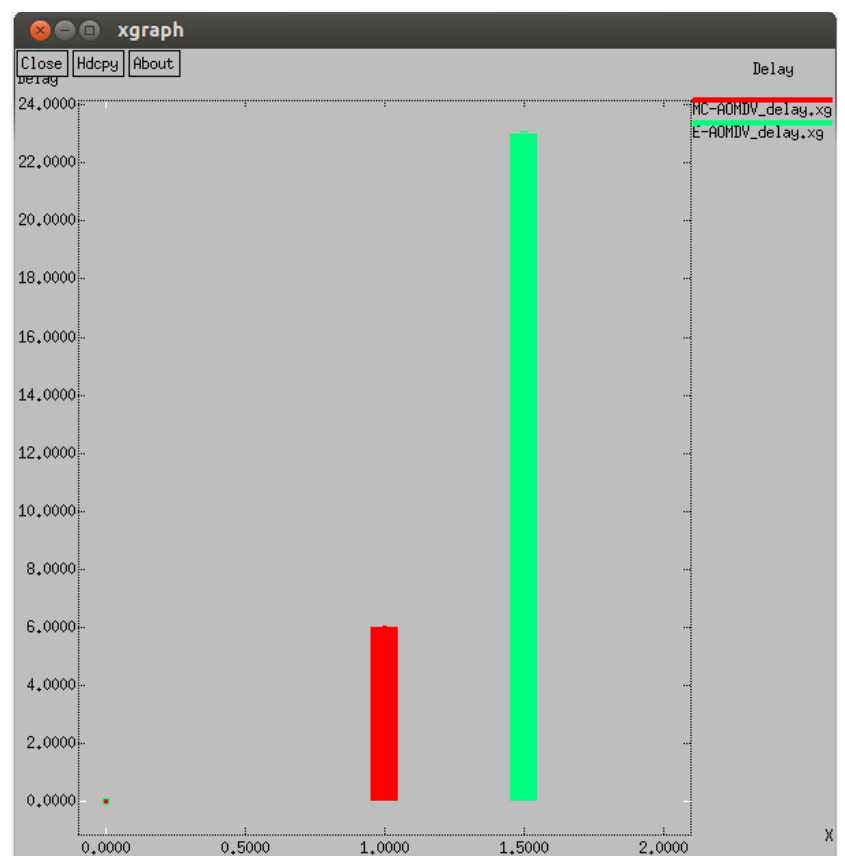

Fig 4: Average latency Comparison of E-AOMDV and MCAOMDV 


\section{ROUTING PACKET OVERHEAD}

The ratio of number of control packets (route request/reply/update/error packets) to the number of data packets is temed as routing packet overhead.

i.e.,

$$
\text { Routing Overhead }=\frac{\text { No.of control packets }}{\text { No.of data packets }}
$$

Whenever the data is sent from any node, it appends a header on the packet. More the routing packets being sent in route discovery phase of the network, more is the routing overhead. The routing overhead for any network should be less. Proposed scheme exhibited less routing overhead of 1.25 as compared to 1.52 exhibited by EAOMDV. Fig 5 shows the comparison of EAOMDV with MC-AOMDV. The routing overhead in case of MC-AOMDV (the proposed scheme) is less than the E-AOMDV.

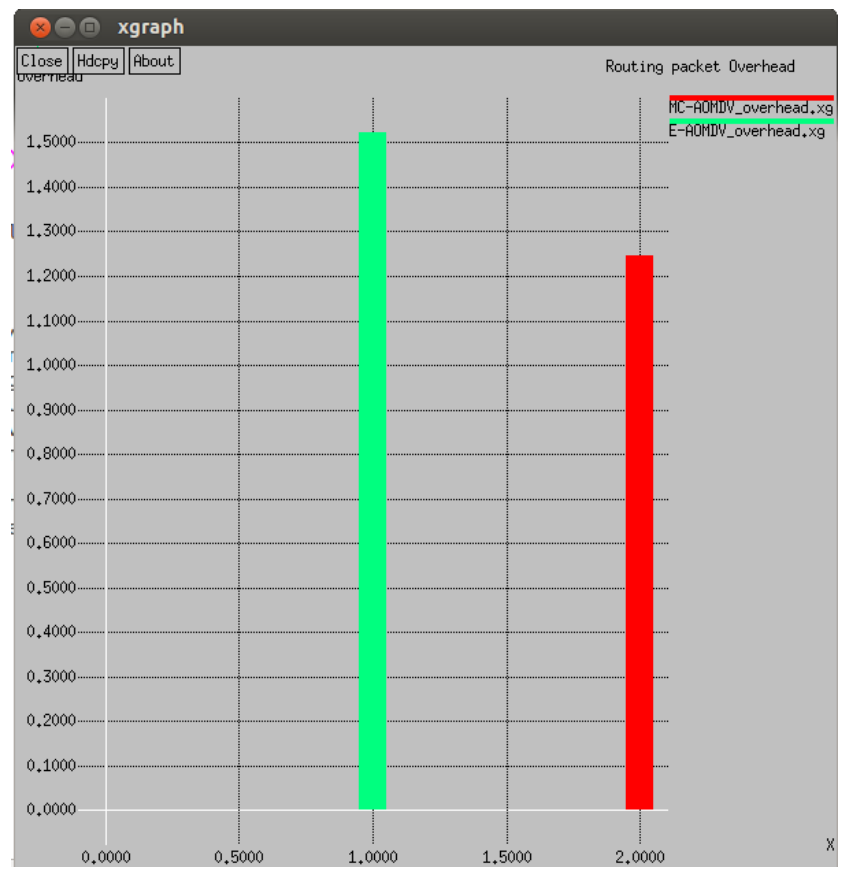

Fig 5: Routing packet overhead comparison of E-AOMDV and MC-AOMDV

\section{CONCLUSIONS}

For the sake of better throughput, load balancing and energy efficiency, multipath routing has been widely studied and used in wireless networks. The proposed approach has been realized using NS2.35 simulator and has been evaluated using different performance metrics i.e. Average energy consumption, Average end-to-end delay, Routing overhead and Throughput. Unlike single path counterparts, multipath on-demand routing protocols can appreciably handle mobility-induced link failures in mobile ad-hoc networks. However, the proposed scheme can be further extended in combination with genetic algorithms to enhance the performance of the network.

\section{REFERENCES}

[1] Aarti, Dr. S.S. Tyagi, "Study of MANET: Characteristics, Challenges, Application and Security Attacks", International Journal of Advanced Research in Computer Science and Software Engineering,Volume 3, Issue 5, May 2013.

[2] Alpesh Chauhan, Prof. B.V. Buddhdev, "AOMDV with improved Energy Function", International Journal of
Advanced Research in Computer Engineering \& Technology, Vol. 2, No. 2, February 2013.

[3] Archana Shukla, Sanjay Sharma, "Queue Length based Load Balancing Technique using with AOMDV Protocol in MAMET", International Journal of Scientific \& Engineering Research (2229-5518), Vol. 4, No. 10, October 2013.

[4] Bhabani Sankar Gouda, Ashish Kumar Dass, K.Lakshmi Narayana "A Comprehensive Performance Analysis of Energy Efficient Routing Protocols in different traffic based Mobile Ad-hoc Networks", 2013.

[5] Bhavna Sharma, Shaila Chugh, Vismay Jain, "Energy Efficient Load Balancing Approach to Improve AOMDV Routing in MANET", 2014 Fourth International Conference on Communication Systems and Network Technologies",IEEE 2014.

[6] Chintan Bhavsar, Mayur M. Vegad, Sunil. A Bakhru, "Performance Comparison of Stability Enhanced AODV and AOMDV Protocols for MAMETs", International Journal of Emerging Technology and Advanced Engineering (2250-2459), Volume 2, No. 5, May 2012.

[7] D. Maheshwari, R. Nedunchezhian, "Balanced Reliable Shortest for AOMDV (BRSR-AOMDV) using TF Mechanism in MANET", International Journal of Computer Technology \& Applications (2229-6093), Vol. 4, No. 6, Nov-Dec 2013

[8] Deeptanoy Ghosh, Poonam Thakur, "A Survey on OnDemand Routing Techniques in MANET", Elixir Comp. Sci. \& Engg. (16219-16224), 2013

[9] Deepthy J., Nishanth Krishnan, "PERFORMANCE IMPROVEMENT OF ENERGY AWARE AND ADAPTIVE ROUTING PROTOCOLS FOR MANETS - A SURVEY” IJRET, eISSN: 2319-1163 | pISSN: 2321-7308 Volume: 03.

[10] Deepti Singh, Birendra Kumar Sharma, Arvind Kumar, "A Survey on challenges in Multipath Routing for Adhoc Netwrorks", International Journal of Emerging Technology and Advanced Engineering, ICADET-14, India (22502459), Volume 4, No. 1, February 2014.

[11] Fan $\mathrm{Zi} \mathrm{Fu}$, Zhang Ling, Wan Xiao Yu, "An Optimized AOMDV Routing Algorithm Based on Self-Adaptive Node Speed in Wireless Sensor Network", 2012 International Workshop on Information and Electronics Engineering (IWIEE 2012).

[12] Geetha.G, N.J.R.Muniraj, "Efficient Energy based Stable Multipath Routing Scheme for MANET", International Journal of Computer Applications (0975 - 8887) Volume 73- No.22, July 2013

[13] Hassanali Nasehi, et al. "Improving Energy Efficiency in MANETs by Multipath Routing", International journal of Wireless \& Mobile Networks (IJWMN), Vol. 5, No. 1, February 2013

[14] Hesham A. Ali, et. al, "Manet Load Balancing Parallel Routing Protocol”, IJCSI, Vol. 9, No. 1, July 2012.

[15] K. Latif, A. Ahmad, et. al, "Divide-and-Rule for Energy Efficient Routing in Wireless Sensor Networks", The $4^{\text {th }}$ International Conference on Ambient Systems, Networks and Technologies (ANT 2013)

[16] M. Bheemalingaiah, M. M. Naidu, D. Sreenivasa Rao3, G.Varaprasad, "Energy Aware Node Disjoint 
MultipathRouting In Mobile Ad Hoc Network", Journal of Theoretical and Applied Information Technology, 20052009

[17] Mahima Chitkara, Mohd. Waseem Ahmad, "Review on MANET: Characteristics, Challenges, Imperatives and Routing Protocols", International Journal of Computer Science and Mobile Computing, Vol. 3, Issue. 2, February 2014.

[18] Manveen Singh Chadha, Rambir Joon, Sandeep, "Simulation and Comparison of AODV, DSR and AOMDV Routing Protocols in MANETs", International Journal of Soft Computing and Engineering (IJSCE) (2231-2307), Vol. 2, No. 3, July 2012.

[19] May Zin Oo, Mazliza Othman, "Performance Comparisons of AOMDV and OLSR Routing Protocols for Mobile Ad Hoc Network", Second International Conference on Computer Engineering and Applications, 2010.

[20] Mina Vajed Khiavi, Shahram Jamali, "Performance Comparison of AODV and AOMDV Routing Protocols in Mobile Ad Hoc Networks", International Research Journal of Applied and Basic Sciences (3277-3285), Vol. 4, No. 11, 2013

[21] Mohamed Tekaya, Nabil Tabbane, Sami Tabbane, "Multipath Routing with Load Balancing and QoS in Adhoc Network", IJCSNS, Vol. 10 No. 8, August 2010.

[22] N. Sumathi, Antony Selvasdoss Thanamani, "ENERGY EFFICIENT BANDWIDTH CONSTRAINED QOS ENABLED MULTIPATH ROUTING FOR MANETs", International Journal of Computing (1727-6209), Vol. 10, No. 3, 2011.

[23] Onkar Singh Bawa, Mr. Supratik Banerjee, "Congestion based Route Discovery AOMDV Protocol", International Journal of Computer Trends and Technology, Vol. 4, No. 1, 2013

[24] P. Periyasamy, Dr. E. Karthikeyan, "Performance Evaluation of AOMDV Protocol Based on Various Scenario and Traffic Patterns", International Journal of Computer Science, Engineering and Applications (IJCSEA), Vol. 1, No. 6, December 2011.

[25] Pinesh A Darji, Mrs. Sumitra Menaria, "An Improvement in AOMDV with Randomization", IJCTA, Vol. 3, No.3, MayJune 2012.

[26] Pinki Nayak, Rekha Agrawal, and Seema Verma, "ENERGY AWARE ROUTING SCHEME FOR MOBILEAD HOC NETWORK USING VARIABLE RANGETRANSMISSION", International Journal of Ad hoc, Sensor \& Ubiquitous Computing (IJASUC) Vol.3, No.4, August 2012.
[27] Priyanaka Bansal, Prof. Anuj K. Gupta, "PERFORMANCE EVALUATION OF AOMDV AND IAOMDV UNDER PRETTY GOOD PRIVACY MODEL", Global Journal of Advanced Engineering Technologies, Vol. 3, Issue 2, 2014.

[28] R. Vinod Kumar, Dr. R.S.D. Wahida Banu, "Load-balancing Approach for AOMDV in Ad-hoc Networks", IJCA, 2010.

[29] Ruchi Gupta, et al, "A Research Paper on Comparison between Energy Efficient Routing Protocol with Energy and Location in MANET", IOSR Journal of Computer Engineering, Vol. 9, No. 4, Mar-Apr 2013.

[30] S. Malini et al., "Traffic based Energy Consumption Analysis of AOMDV Protocol in Mobile Ad Hoc Netwrork", International Journal of Computer Applications (0975-8887), Volume 14- No. 6, February 2011

[31] S. R. Biradar, et. al., "Performance Evaluation and Comparison of AODV and AOMDV", International Journal on Computer Science and Engineering (373-377), Vol. 2, No. 2, 2010.

[32] Sanghitra Bhattacharjee, Subhansu Bandyopadhyay, "Lifetime maximizing dynamic energy efficient routing protocol for multi hop wireless networks", Simulation Modelling Practice and Theory, 2013.

[33] Sanjeev Sharma and Sanjay Singh, "A Survey Of Routing Protocols And Geographic Routing Protocol Using GPS In Manet", Volume 3, No. 12, December 2012, Journal of Global Research in Computer Science.

[34] Sasan Adibi, Shervin Erfani, "A Multipath Routing Survey for Mobile Ad-Hoc Networks", IEEE 2005.

[35] Sujatha P Terdal, Dr. V.D.Mytri, Dr. A.Damodaram, "A Load Aware Routing Mechanism for Improving Energy Efficiency in Mobile Ad Hoc Networks", International Journal of Computer Applications (0975-8887), Vol. 10No. 3, November 2010.

[36] Vinay Kumar, (Dr.) C.Ram.Singla, "Enhanced Ad Hoc On Demand MultipathDistance Vector For Manets", Journal of Theoretical and Applied Information Technology 20th August 2014. Vol. 66 No.2

[37] Vipul Maheshwari, Shrikant Jadav, "Survey on MANET Routing Protocol and Multipath Extension in AODV", International Journal of Applied Information Systems (IJAIS) - ISSN : 2249-0868Foundation of Computer Science FCS, New York, USA, Volume 2- No.4, May 2012

[38] Wesam AlMobaideen," SPDA: Stability Based Partially Disjoint AOMDV", European Journal of Scientific Research, Vol. 27, No.3, 2009.

[39] Yumei Liu, Lili Guo, et. al., "Energy Efficient on-demand Multipath Routing Protocol for Multi-hop Ad Hoc Networks", IEEE 2008. 